Article

\title{
Hydrophobic Modification of Magnesium Hydroxide Coating Deposited Cathodically on Magnesium Alloy and its Corrosion Protection
}

\author{
Yongjun Zhang ${ }^{1, *}$, Hui Cao ${ }^{1}$, Hanxiong Huang ${ }^{1}$ and Zhiping Wang ${ }^{2, *}$ \\ 1 School of Mechanical and Automotive Engineering, South China University of Technology, \\ Guangzhou 510640, China \\ 2 Guangdong Provincial Engineering Center of Topical Precise Drug Delivery System, \\ Guangdong Pharmaceutical University, Guangzhou 510006, China \\ * Correspondence: zhangyj@scut.edu.cn (Y.Z.); wzping-jshb@gdpu.edu.cn (Z.W.); \\ Tel.: +86-20-87111032 (Y.Z.); +86-20-39352129 (Z.W.)
}

Received: 2 January 2019; Accepted: 18 February 2019; Published: 29 July 2019

\begin{abstract}
In this paper, hydrophobic modification of magnesium hydroxide film prepared by cathodic deposition on ME20M Mg alloy was achieved with ethanol solution of stearic acid. The effects of solution concentration, temperature and treatment time on the surface wettability were investigated. The corrosion behaviors of typical samples were evaluated by droplet test, total immersion corrosion test as well as PDP (Potentiodynamic Polarization) and EIS (Electrochemical Impedance Spectroscopy) techniques, respectively. Adhesion of coatings to the substrate were examined by tape test. The surface morphology and chemical composition of typical coatings were characterized by SEM, FTIR and XRD respectively. The results indicate that the superhydrophobic surface with $151.5^{\circ}$ water static contact angle and $1^{\circ}$ sliding angle could be fabricated successfully by simple immersion in a low-concentration stearic acid solution for a short period. Compared with blank sample, the composite surface modification based on cathodic deposition (CD) plus hydrophobic treatment (HT) extended the discoloration time in droplet tests by nearly 25 fold, decreased the $i_{\text {corr }}$ (corrosion current density) by three orders of magnitude, and increased the characteristic impedance modulus at $0.1 \mathrm{~Hz}$ by nearly four orders of magnitude, indicating that the $\mathrm{CD} / \mathrm{HT}$ composite surface modification could enhance the corrosion resistance of $\mathrm{Mg}$ alloy significantly.
\end{abstract}

Keywords: magnesium alloy; cathodic deposition; magnesium hydroxide coating; stearic acid; superhydrophobicity; corrosion resistance

\section{Introduction}

Magnesium $(\mathrm{Mg})$ alloys, which are known as 21st century green engineering materials, are inexpensive and abundant and have the advantages of low density, good recyclability, strong electromagnetic shielding ability, and good biocompatibility [1,2]. Therefore, they are widely employed in the aerospace, vehicle, IT, electronics, and medical device industries [3]. Compared with other metal implant materials used clinically, $\mathrm{Mg}$ alloys have the following outstanding advantages: (a) excellent biocompatibility, mechanical compatibility and biodegradability; (b) a density similar to that of human bones; and (c) high specific strength and specific stiffness. However, $\mathrm{Mg}$ alloys have poor corrosion resistance and especially in the corrosive media containing chloride ion because of the destruction of the native oxide film at their surface. Therefore, surface modifications have been one of the most effective and economical techniques used to control the corrosion of $\mathrm{Mg}$ alloys.

Superhydrophobic surfaces have been widely studied and used in self-cleaning, antifreezing and low-viscosity applications, etc. The hydrophobicity can also improve the corrosion protection 
of metal materials significantly [4-6]. In order to improve the corrosion resistance of $\mathrm{Mg}$ alloys, it's important to study their hydrophobic treatment (HT) [7]. Surface wettability, which refers to the ability of water to spread on solid surfaces, is an important indicator for evaluating hydrophobic properties. Generally, a surface with a water contact angle less than $90^{\circ}$ is considered hydrophilic; a surface with a water contact angle between $90^{\circ}$ and $150^{\circ}$ is considered hydrophobic; and a surface with a water contact angle exceeding $150^{\circ}$ is considered superhydrophobic [8,9]. The surface wettability is determined by its chemical composition and microstructure [10,11]. Major approaches to preparing superhydrophobic surfaces have included hydrothermal synthesis [12], sol-gel method [13], spray-coating [14], electroplating [15], and chemical etching [16], etc.

At present, superhydrophobic films/coatings at the surfaces of $\mathrm{Mg}$ alloys are generally obtained by the following steps: (a) manufacturing a rough surface structure at micro/nano scale and (b) reducing the surface free energy with low surface energy reagents, such as stearic acid, myristic acid and lauric acid [17-19]. In our previous work [20], in situ-grown, mesh-like, and rough $\mathrm{Mg}(\mathrm{OH})_{2}$ coatings were synthesized by cathodic deposition on pure Mg. It's demonstrated that they could improve the corrosion resistance of pure $\mathrm{Mg}$ metal to some extent. In order to enhance the corrosion protection of $\mathrm{Mg}(\mathrm{OH})_{2}$ coatings prepared by cathodic deposition further, an ingenious method of modifying them to obtain superhydrophobicity is introduced and discussed in this paper.

In order to manufacture the Mg alloys with superhydrophobic surfaces, scholars have made fruitful endeavours. For example, Zhong et al. [21] reported the successful fabrication of superhydrophobic coatings (myristic calcium) on AZ31 Mg alloy with a contact angle of $157^{\circ}$ via a simple one-step electrodeposition method. Zhang et al. [22] electrodeposited a hydrophobic calcium stearate coating onto an anodized $\mathrm{Mg}$ to decrease the degradation rate of the substrate in simulated body fluid. Gao et al. [23] fabricated a hierarchically structured szaibelyite film on AZ31 Mg alloy by means of template-free hydrothermal synthesis, and then modified the surface with fluoroalkyl silane (FAS); the resulting film became superhydrophobic, with a contact angle of $166^{\circ}$ and a rolling angle of $5^{\circ}$. Liu et al. [24] modified $\mathrm{Mg}$ alloy by a composite process of nickel plating and stearic acid treatment to obtain superhydrophobic surfaces. Zhu et al. [25] found that superhydrophobic surfaces with a contact angle of $154^{\circ}$ and a rolling angle of $6^{\circ}$ were successfully synthesized on $\mathrm{Mg}$ alloy by a serial process of hydrochloric acid etching, ammonia immersion and subsequent stearic acid treatment. Han et al. [26] combined chemical etching with nichel sulfate and treatment with stearic acid to induce superhydrophobicity. Ishizaki and Sakamoto [27] fabricated color-tuned superhydrophobic surfaces by a chemical-free immersion process in ultrapure water and modification with n-octadecyltrimethoxysilane.

In this study, based on $\mathrm{Mg}(\mathrm{OH})_{2}$ coatings prepared by cathodic deposition, superhydrophobic surfaces were manufactured on ME20M Mg alloy using environmentally friendly, low-cost reagents. The effects of typical surface treatment, especially CD/HT composite modification, on the wettability and corrosion resistance of $\mathrm{Mg}$ alloy were investigated.

\section{Materials and Methods}

As-rolling ME20M Mg alloy sheet was employed in this paper. Its main chemical composition was as follows: Mn 1.5\%, Ce 0.3\%, Al 0.01\%, Fe 0.01\%, Si 0.02\%, La $0.003 \%$, and balance Mg. The sizes of samples were $40 \mathrm{~mm} \times 40 \mathrm{~mm} \times 3 \mathrm{~mm}$. Their surfaces were ground with SiC paper from $240 \mathrm{up}$ to 1000 grit, rinsed with distilled water, ultrasonically degreased in ethyl alcohol and dried under a stream of hot air. Afterwards, the samples were measured with a Vernier caliper and weighed on an electronic analysis balance (B Sartorius, Göttingen, Germany).

$\mathrm{Mg}(\mathrm{OH})_{2}$ coating was fabricated by cathodic deposition in an aqueous solution containing $25 \mathrm{~g} / \mathrm{L}$ $\mathrm{Mg}(\mathrm{NO})_{3}$ and $1.5 \mathrm{~mL} / \mathrm{L} \mathrm{H}_{2} \mathrm{O}_{2}$ at $37 \pm 1{ }^{\circ} \mathrm{C}$ employing a two-electrode system, where the distance between sample (working electrode) /graphite flake (counter electrode) was $3.0 \mathrm{~cm}$. A programmable power supply (IT7000, ITECH, Zhubei, Taiwan, China) was employed and a pulse current was applied. The pulsing parameters were as follows: peak current density $1.6 \mathrm{~mA} / \mathrm{cm}^{2}$, total power-on time $2700 \mathrm{~s}$ 
with a cycle of $5 \mathrm{~s}$ conduction and $0.03 \mathrm{~s}$ off. After cathodic deposition, the samples were rinsed in distilled water and dried using a stream of hot air. Then they were immersed into the ethanol solution of stearic acid for a certain time to modify. During immersion, the $V / S$ ratio was kept to $5 \mathrm{~mL} / \mathrm{cm}^{2}$, where $V$ solution volume, $\mathrm{S}$ surface area of sample.

A contact angle instrument (OCA35, Dataphysics, Stuttgart, Germany) was employed. The static contact angle (SCA, advancing contact angles only) and sliding angle (SA) of distilled water droplets $(5 \mu \mathrm{L})$ were measured five times at selected areas at the surface of sample. During test, the temperature and relative humidity were controlled at $25^{\circ} \mathrm{C}$ and $65 \% \mathrm{RH}$, respectively.

Adhesion between coating and $\mathrm{Mg}$ substrate was assessed by tape test method. The classification of the adhesion test results according to ASTM D3359-2009 [28] was presented in Table 1.

Table 1. Classification of adhesion test results.

\begin{tabular}{ccccccc}
\hline Percent Area Removed & $\mathbf{0 \%}$ & Less Than $\mathbf{5 \%}$ & $\mathbf{5 \%} \mathbf{- 1 5 \%}$ & $\mathbf{1 5 \%} \mathbf{- 3 5 \%}$ & $\mathbf{3 5 \% - 6 5 \%}$ & Greater Than $\mathbf{6 5 \%}$ \\
\hline Classification & $5 \mathrm{~B}$ & $4 \mathrm{~B}$ & $3 \mathrm{~B}$ & $2 \mathrm{~B}$ & $1 \mathrm{~B}$ & $0 \mathrm{~B}$ \\
\hline
\end{tabular}

The corrosion resistance of typical samples was studied by droplet test, total immersion corrosion test as well as PDP and EIS techniques. In the droplet test, $50 \mathrm{mg} / \mathrm{L} \mathrm{KMnO}_{4}$ was used as the indicator, and $10 \mathrm{~mL} / \mathrm{L} \mathrm{HNO}_{3}(65 \mathrm{wt} \%)$ as the etchant. Five points were picked out at each typical surface to evaluate the average discoloration time. The total immersion corrosion test was performed at $37^{\circ} \mathrm{C}$ for $48 \mathrm{~h}$. The average corrosion rate was calculated by Equation (1):

$$
v=1000 \times\left(m_{0}-m_{1}\right) / S
$$

where $v$ is the average corrosion rate, $\mathrm{mg} / \mathrm{cm}^{2} ; m_{0}$ is the original mass of the sample before the test, $\mathrm{g}$; $m_{1}$ is the mass of the sample after the test was finished and the corrosion products were removed, $\mathrm{g}$; $S$ is the working surface area of the sample, $\mathrm{cm}^{2}$.

Data on PDP curves and EIS were collected with an electrochemical workstation (Chenhua CHI604, Shanghai, China) employing a three-electrode system with sample as WE (Working Electrodes), saturated calomel electrode (SCE) as RE (Reference Electrode) and Pt sheet as AE (Auxiliary Electrode). The PDP tests were taken at a scanning rate of $0.5 \mathrm{mV} / \mathrm{s}$. The EIS tests were performed at $E_{\text {corr }}$ from $100 \mathrm{kHz}$ to $10 \mathrm{mHz}$ at five frequency points per decade with $10 \mathrm{mV} \mathrm{rms} \mathrm{amplitude} \mathrm{sinusoidal}$ perturbing signal.

In the tests of total immersion corrosion, PDP and EIS, $3.5 \mathrm{wt} . \% \mathrm{NaCl}$ solution aerated was employed as testing medium. The ratio of medium volume to working area of sample was $200 \mathrm{~mL} / \mathrm{cm}^{2}$. The $\mathrm{pH}$ value of $\mathrm{NaCl}$ solution was adjusted to 7.00 using diluted $\mathrm{NaOH}$ or $\mathrm{HCl}$ solution. While electrochemical tests controlled remotely by a PC were running, the solution remained quiescent and its temperature was maintained at $30 \pm 1{ }^{\circ} \mathrm{C}$.

The micromorphologies of typical samples were observed by scanning electron microscopy (SEM, Quanta 650, FEI, Hillsboro, OR, USA). The chemical compositions were examined with a Fourier transform infrared spectrometer (FT-IR; VERTEX 33, Bruker, Karlsruhe, Germany) using the $\mathrm{KBr}$ technique and X-ray diffractometer (XRD; D8 Advance, Bruker, Karlsruhe, Germany).

In this paper, all the solutions were prepared by using analytical grade reagents and distilled water. All the potentials were on $\operatorname{SCE}$ scale $\left(E_{\mathrm{SCE}}=0.2438 \mathrm{~V}\right.$ vs $\left.E_{\mathrm{NHE}}\right)$. 


\section{Results and Discussion}

\subsection{Effects of Modifying Parameters}

\subsubsection{Concentration of stearic acid}

In order to investigate the effect of the concentrations of stearic acid on the surface wettability of $\mathrm{Mg}$ alloy substrate, after preparing $\mathrm{Mg}(\mathrm{OH})_{2}$ coating by cathodic deposition, the samples were immersed into ethanol solutions of stearic acid at $80^{\circ} \mathrm{C}$ for $1 \mathrm{~h}$. The test results of SCA and SA (Figure 1) showed that with an increase in the content of stearic acid, the SCA first increased and then decreased almost linearly. When the concentration was $5 \mathrm{~g} / \mathrm{L}$, the SCA reached its maximum value $151.5^{\circ}$. With a further increase in the concentration, the surfaces of the samples were hydrophobic yet. But the SCAs decreased to less than $150^{\circ}$. It was confirmed that the $\mathrm{Mg}(\mathrm{OH})_{2}$ coating prepared by cathodic deposition could obtain superhydrophobicity after immersion in a low concentration stearic acid solution for a short period. All SAs were $1^{\circ}$, indicating that the concentration of stearic acid had no effect on the SAs in the concentration window studied and within the accuracy of testing instrument. Based on the above results, for the purpose of hydrophobic treatment of $\mathrm{Mg}(\mathrm{OH})_{2}$ coatings prepared by cathodic deposition, the optimal concentration of stearic acid was $5 \mathrm{~g} / \mathrm{L}$ while using anhydrous ethanol as solvent.

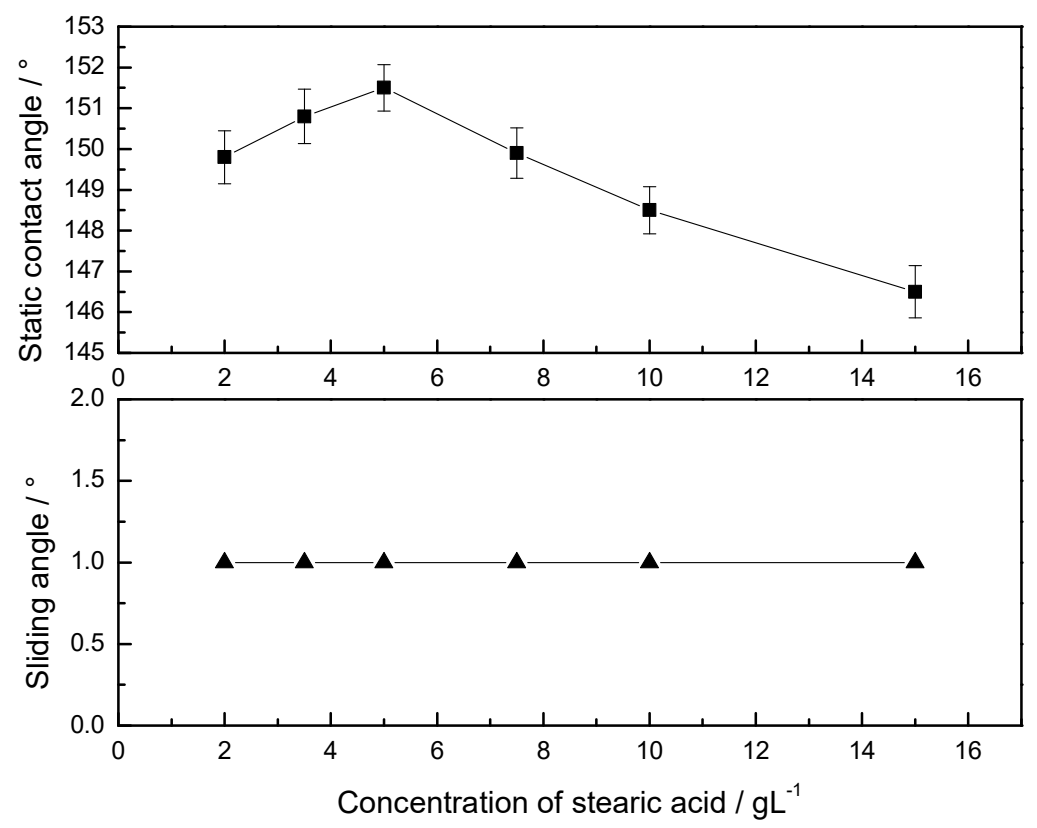

Figure 1. Variations of SCAs, SAs with the concentration of stearic acid.

\subsubsection{Solution Temperature}

Modification parameters were designed as follows in the single factor experiment: anhydrous ethanol as solvent, content of stearic acid $5 \mathrm{~g} / \mathrm{L}$, immersion time $1 \mathrm{~h}$, solution temperature $\mathrm{T}$ as single variable. Prior to immersion modification, a thin coating of $\mathrm{Mg}(\mathrm{OH})_{2}$ was prefabricated at the surface of each sample by cathodic deposition. The variations of their surface hydrophobicity were presented in Figure 2. It's obvious that the SCAs were affected very slightly. In the temperature window studied, they almost remained $150.0^{\circ}$ stable. However, the $T$ affected the SA strongly: the higher the former, the smaller the latter. The linear relationship between them was clearly evident and the fitted equation was as follows: $\mathrm{SA}=-0.1 \mathrm{~T}+9$. Hence, the optimal solution temperature was $80^{\circ} \mathrm{C}$. 

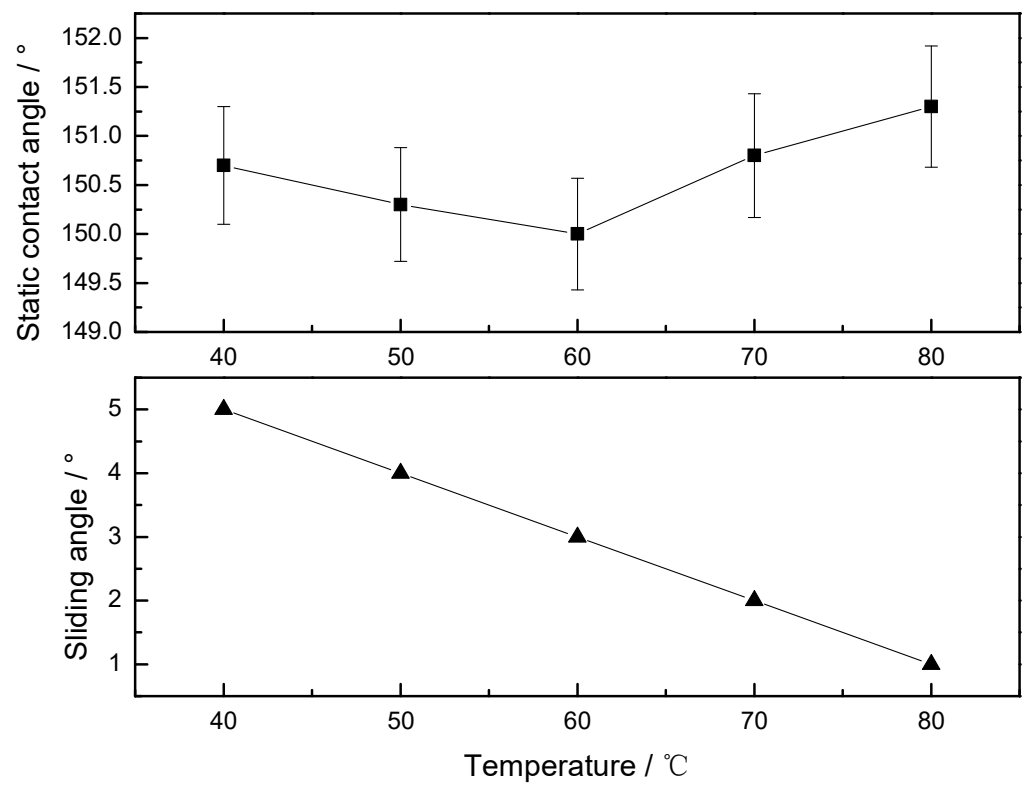

Figure 2. Variations of SCAs, SAs with solution temperature.

\subsubsection{Treatment Time}

Immersion time $t$ was chosen as variable in the single factor experiment under the following conditions: $5 \mathrm{~g} / \mathrm{L}$ stearic acid using anhydrous ethanol as solvent, solution temperature $80^{\circ} \mathrm{C}$. Test results shown in Figure 3 demonstrated that the SCA increased rapidly at the initial stage but then decreased slowly with the prolongation of time. The SCA increased almost linearly with the $t$. It took the samples only 10 minutes to realize surface hydrophobization. The peak value of $151.5^{\circ}$ appeared after $1 \mathrm{~h}$ of immersion. When $t$ was extended further, the SCA decreased slightly but remained greater than $150.0^{\circ}$. For the SA, it decreased linearly with time from $5^{\circ}$ to $1^{\circ}$ while $t$ was less than $1 \mathrm{~h}$. But it's not affected by time any longer and stabilized at the level of $1^{\circ}$ while $t$ exceeded $1 \mathrm{~h}$. The above relationship between wettability and $t$ should be attributed to the different proceeding of self-assembly.
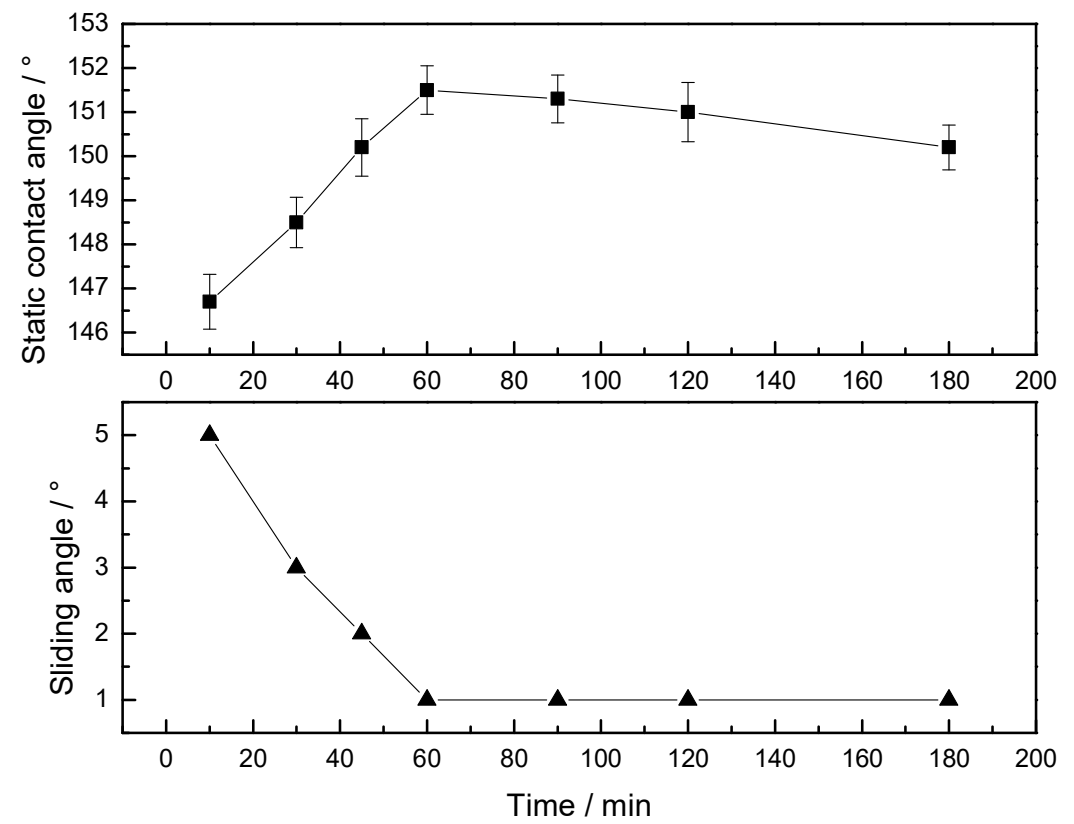

Figure 3. Relationship between SCAs, SAs and immersion time. 


\subsection{Corrosion Protection}

The Mg alloy sample ground only was denoted as Blank sample. The sample modified by stearic acid further was denoted as Blank + HT (Hydrophobic Treatment) sample. The sample prepared by cathodic deposition was denoted as CD sample. The CD sample modified by stearic acid further was denoted as CD + HT sample. The hydrophobic treatment was performed under the optimal conditions: ethanol solution of stearic acid solution of $5 \mathrm{~g} / \mathrm{L}$ at $80^{\circ} \mathrm{C}$ for $1 \mathrm{~h}$.

\subsubsection{Droplet Test}

The results of droplet test shown in Table 2 indicated that hydrophobic treatment could effectively improve the corrosion resistance of the $\mathrm{Mg}$ alloy, including the Blank sample. For the Blank sample, the discoloration time of corrosive droplet increased more than twofold. However, its increase was far less than those observed for the CD and CD + HT samples. The discoloration time for the CD + HT sample was twice that of the CD sample and was approximately eight times that of the Blank + HT sample. Obviously, surface modification of $\mathrm{Mg}(\mathrm{OH})_{2}$ coatings prepared by cathodic deposition with stearic acid could improve the corrosion resistance of $\mathrm{Mg}$ alloy significantly.

Table 2. Discoloration time of corrosive droplets on typical samples.

\begin{tabular}{ccccc}
\hline Sample & Blank & CD & Blank + HT & CD + HT \\
\hline Discoloration time, s & 40 & 550 & 134 & 1037 \\
\hline
\end{tabular}

\subsubsection{Total Immersion Corrosion Test}

Figure 4 presented the results of total immersion corrosion test in which $3.5 \mathrm{wt} \% \mathrm{NaCl}$ solution with $\mathrm{pH} 7.0$ was employed as testing medium at $37^{\circ} \mathrm{C}$ for $48 \mathrm{~h}$. Although single CD or HT could inhibit the corrosion of $\mathrm{Mg}$ alloy substrate to some extent, $\mathrm{CD} / \mathrm{HT}$ composite modification was the most effective. Actually, the obtained average corrosion rate $v$ corresponding to CD + HT sample was $1.2 \mathrm{mg} / \mathrm{cm}^{2}$, which decreased by $52 \%$ compared with the Blank sample. The above results further confirmed that surface modification of the $\mathrm{Mg}(\mathrm{OH})_{2}$ coatings prepared by cathodic deposition could improve the corrosion resistance of $\mathrm{Mg}$ alloy significantly.

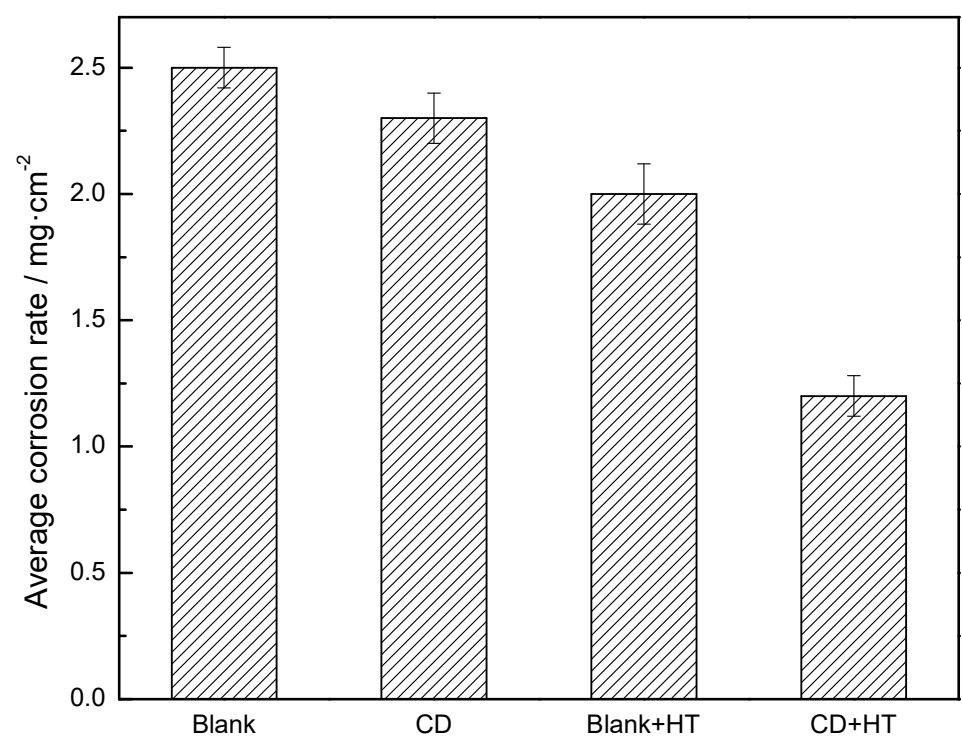

Figure 4. Average corrosion rate of $\mathrm{Mg}$ alloy samples in $3.5 \mathrm{wt} \% \mathrm{NaCl}$ solution with $\mathrm{pH} 7.0$ at $37^{\circ} \mathrm{C}$ for $48 \mathrm{~h}$. 


\subsubsection{Electrochemical Measurements}

Figure 5 showed the PDP curves of the typical samples. Table 3 listed the fitting results of the corrosion potential $\left(E_{\text {corr }}\right)$ and corrosion current density $\left(i_{\text {corr }}\right)$. In terms of $i_{\text {corr }}$, the variation trend of corrosion resistance was basically consistent with the results from the droplet test. The $i_{\text {corr }}$ of the CD + HT sample was $6.2955 \times 10^{-3} \mu \mathrm{A} / \mathrm{cm}^{2}$, which was three orders of magnitude smaller than those of the Blank and CD samples. In addition, even for the Blank sample, its $i_{\text {corr }}$ decreased to approximately $45 \%$ after hydrophobic treatment. Noticeably, the decrease was far less than that of the CD + HT sample.

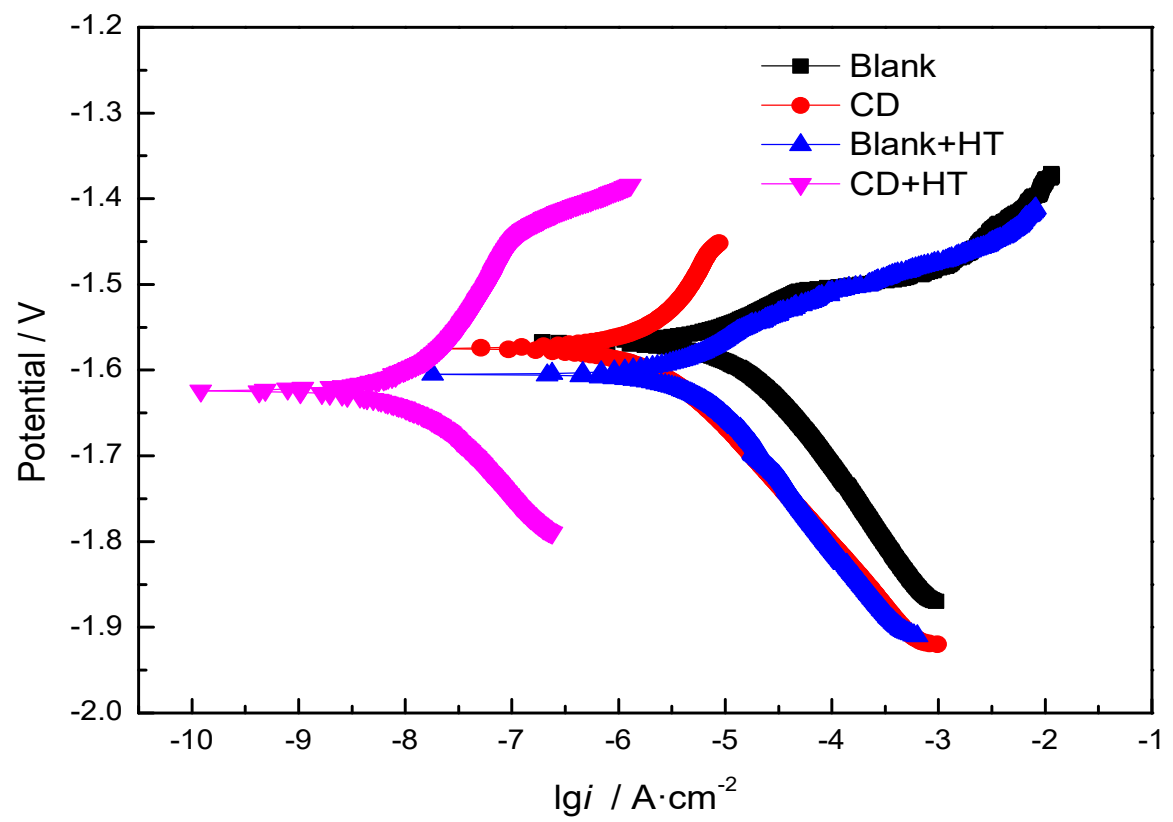

Figure 5. Potentiodynamic polarization (PDP) curves for typical samples in $3.5 \mathrm{wt} \% \mathrm{NaCl}$ solutions.

Table 3. Relevant electrochemical parameters from the polarization curves.

\begin{tabular}{ccc}
\hline Sample & $\boldsymbol{E}_{\text {corr }}(\mathrm{V})$ & $\boldsymbol{i}_{\text {corr }}\left(\boldsymbol{\mu A} / \mathbf{c m}^{2}\right)$ \\
\hline Blank & -1.5675 & 5.3660 \\
CD & -1.5748 & 2.5588 \\
Blank + HT & -1.6049 & 2.4051 \\
CD + HT & -1.6240 & $6.2955 \times 10^{-3}$ \\
\hline
\end{tabular}

Figure 6 presented the Nyquist plots for typical samples from the EIS tests. Table 4 showed the characteristic impedance modulus at $0.1 \mathrm{~Hz},|\mathrm{Z}|_{0.1 \mathrm{~Hz}}$. The spectra of the samples other than the Blank sample showed typical double-arc structures. In addition, the radius of the impedance arc of the CD + HT sample was much larger than that of the other samples. The $|Z|_{0.1 \mathrm{~Hz}}$ of the CD or Blank + HT samples were increased by only one order of magnitude respectively compared with that of the Blank sample. However, the $|Z|_{0.1 \mathrm{~Hz}}$ of the $\mathrm{CD}+\mathrm{HT}$ sample, $1.45 \times 10^{6} \Omega \cdot \mathrm{cm}^{2}$, was three orders of magnitude greater than those of the CD and Blank + HT samples and four orders of magnitude greater than that of the Blank sample. These results were consistent with the droplet and PDP test results and confirmed further that surface modification of the $\mathrm{Mg}(\mathrm{OH})_{2}$ coatings prepared by cathodic deposition could improve the corrosion resistance of $\mathrm{Mg}$ alloy significantly. 


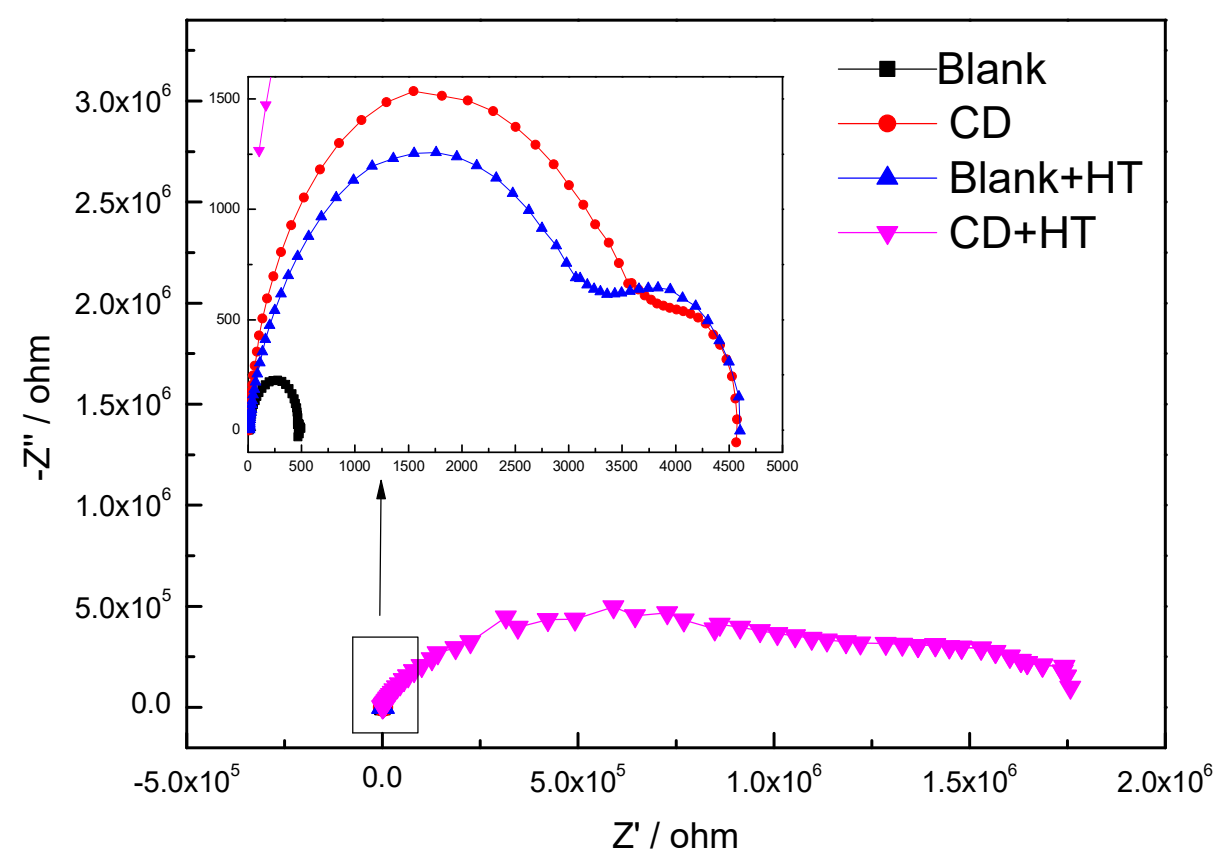

Figure 6. Nyquist plots for typical samples in $3.5 \mathrm{wt} \% \mathrm{NaCl}$ solution.

Table 4. $|Z|_{0.1 \mathrm{~Hz}}$ for typical samples, $\Omega \cdot \mathrm{cm}^{2}$

\begin{tabular}{ccccc}
\hline Sample & Blank & CD & Blank + HT & CD + HT \\
\hline$|Z|_{0.1 \mathrm{~Hz}}$ & $4.66 \times 10^{2}$ & $4.14 \times 10^{3}$ & $3.18 \times 10^{3}$ & $1.45 \times 10^{6}$ \\
\hline
\end{tabular}

\subsection{Surface Wettability}

Figure 7 illustrated distilled water droplets on the surfaces of typical samples and presented the SCA results. The SCA of the Blank sample was only $88.0^{\circ}$ (Figure 7a), implying that metallographic grinding treatment could produce hydrophilic surface on $\mathrm{Mg}$ alloy substrate. The $\mathrm{Mg}(\mathrm{OH})_{2}$ coating prepared by cathodic deposition was superhydrophilic, with a contact angle of barely $15.5^{\circ}$ (Figure $7 \mathrm{~b}$ ). A comparison between Figure 7a,c indicated that even the Blank sample could obtain hydrophobicity after simple immersion in ethanol solution of stearic acid. However, the modification effect based on Blank sample was far less than that based on CD sample. After modification by stearic acid, the surface of CD sample became superhydrophobic with a $151.5^{\circ}$ SCA (Figure $7 \mathrm{~d}$ ), which was nearly $31 \%$ higher than that of the Blank + HT sample and almost 8.8 times higher than that before hydrophobic treatment.

According to the Cassie-Baxter equation (Equation (2)):

$$
\cos \theta_{\mathrm{r}}=f_{1} \cos \theta_{1}-f_{2}
$$

where $f_{1}$ is the contact area fraction of the water droplet with the solid surface, $f_{2}$ is the contact area fraction of the water droplet with the air, and $f_{1}+f_{2}=1 . \theta_{\mathrm{r}}$ is the contact angle of the water droplet on the rough surface. $\theta_{1}$ is the intrinsic contact angle of the water droplet on the smooth surface.

Herein, for the $C D+\mathrm{HT}$ sample, $\theta_{\mathrm{r}}$ was $151.5^{\circ}$ and $\theta_{1}$ was the contact angle of the water droplet on the surface of the smooth $\mathrm{Mg}$ alloy modified with stearic acid, that is, the surface contact angle of the Blank + HT sample, namely $116^{\circ}$. After calculation, $f_{1}$ was $21.6 \%$ and $f_{2}$ was $78.4 \%$, which demonstrated that the air bubbles in the rough structure of the superhydrophobic surface could effectively inhibit the direct contact of the water droplets with the substrate. 

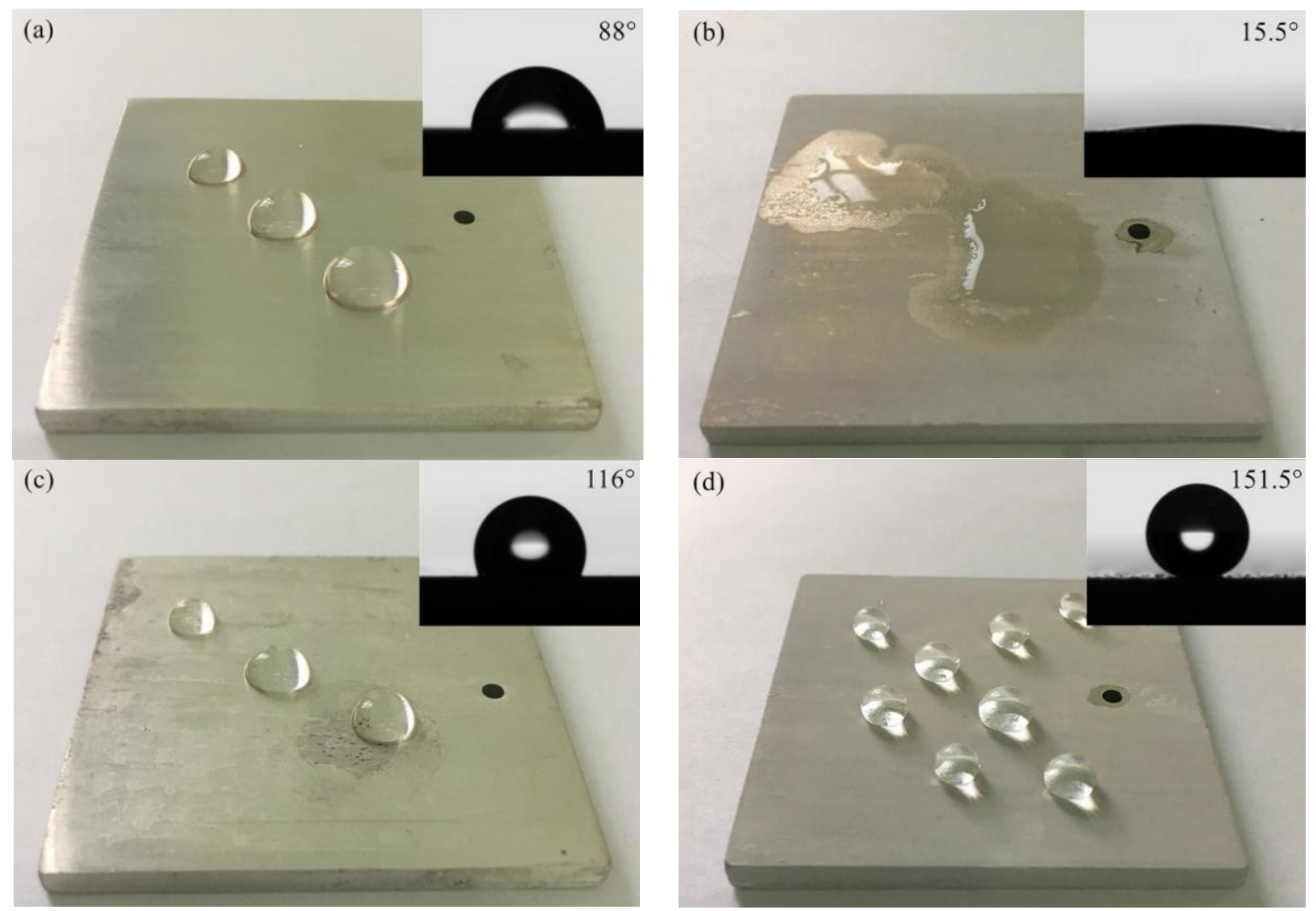

Figure 7. Images of distilled water droplets and the contact angle results (inset) of typical samples:

(a) Blank: $88^{\circ}$; (b) CD: $15.5^{\circ}$; (c) Blank + HT: $116^{\circ}$; (d) CD + HT: $151.5^{\circ}$.

\subsection{Coating Adhesion}

Table 5 listed the adhesion test results according to ASTM D3359-2009 for the CD and CD + HT samples. It's obvious that the coating/substrate adhesion levels under two treatment conditions were both $5 \mathrm{~B}$, implying that after hydrophobic treatment, the $\mathrm{Mg}(\mathrm{OH})_{2}$ coating still closely adhered to the substrate.

Table 5. Adhesion test results of typical samples.

\begin{tabular}{cccc}
\hline Sample & Ratio of Film Spalling & Adhesion Level & Image of Film Spalling \\
\hline $\mathrm{CD}$ & $0 \%$ & $5 \mathrm{~B}$ & \\
\hline & & & \\
$\mathrm{CD}+\mathrm{HT}$ & & & \\
& & & \\
& & & \\
& & & \\
\end{tabular}




\subsection{Micromorphology}

Figure 8 showed the surface micromorphologies of the CD and CD + HT samples at different magnifications. As illustrated, the surface the CD (Figure $8 \mathrm{a}, \mathrm{b}$ ) sample had complex blade-like micro-structure, similar to that of the CD + HT sample (Figure 8c,d), with different micro/nano scale sizes ranging from $300 \mathrm{~nm}$ to $3 \mu \mathrm{m}$. This kind of structure could create a large number of voids with low surface energy reagents and effectively capture air to form air bubbles, which could inhibit the surface to contact with water and corrosive fluids. Therefore, the surface of the Mg substrate was modified to obtain superhydrophobictiy and its corrosion resistance was improved.

Note that even though they have similar micromorphologies, the CD sample was hydrophilic, whereas the $C D+H T$ sample was superhydrophobic according to the results in Section 3.3. The difference was that the surface of the CD sample had not been treated with stearic acid and therefore did not exhibit superhydrophobicity. In addition, even though it had been treated with stearic acid, the Blank sample was not superhydrophobic because of the absence of cathodic deposition. Based on the above results, it was concluded that only CD/HT composite surface modification could realize surface superhydrophobization.
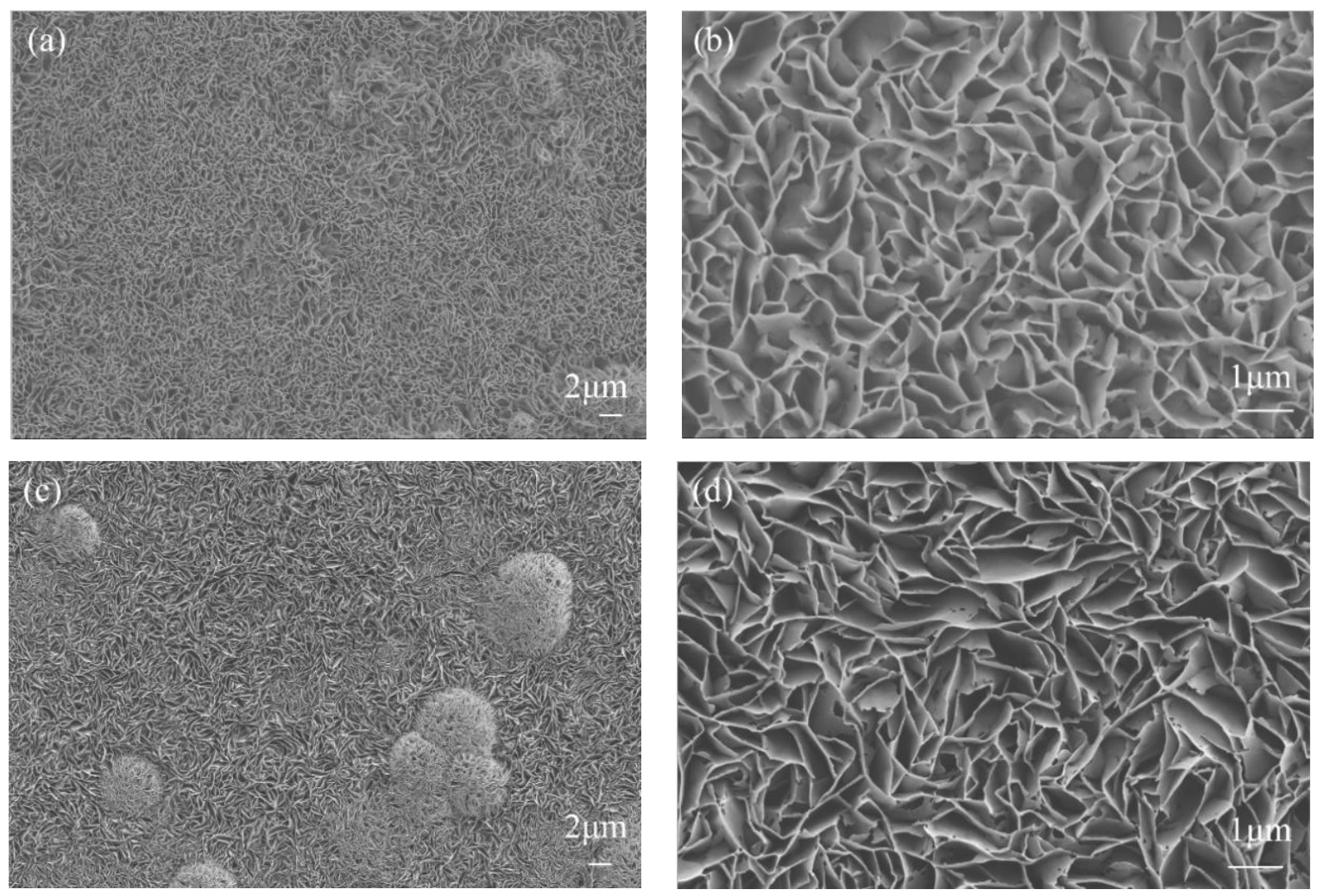

Figure 8. Surface scanning electron microscopy (SEM) images of typical samples: (a) CD: 2,000×; (b) CD: 10,000×; (c) CD + HT: 2,000×; (d) CD + HT: 10,000×.

\subsection{Chemical Composition}

Figure 9 showed the XRD results for the typical Mg alloy samples. It's obvious that the main phase composition of coatings was $\mathrm{Mg}(\mathrm{OH})_{2}$. Compared with the XRD pattern of the CD sample, that of the CD + HT sample did not show a new diffraction peak, which should be attributed to the trace and/or amorphous new products.

To further characterize the chemical composition of the coatings, infrared spectrophotometer was employed. The results shown in Figure 10 demonstrated that new absorption peaks at 2920 and $2848 \mathrm{~cm}^{-1}$ and indicated the presence of $-\mathrm{CH}_{2}-$ based on the standard infrared spectrum of 
stearic acid. These peaks suggested that stearic acid had reacted with $\mathrm{Mg}(\mathrm{OH})_{2}$ coating prepared by cathodic deposition.

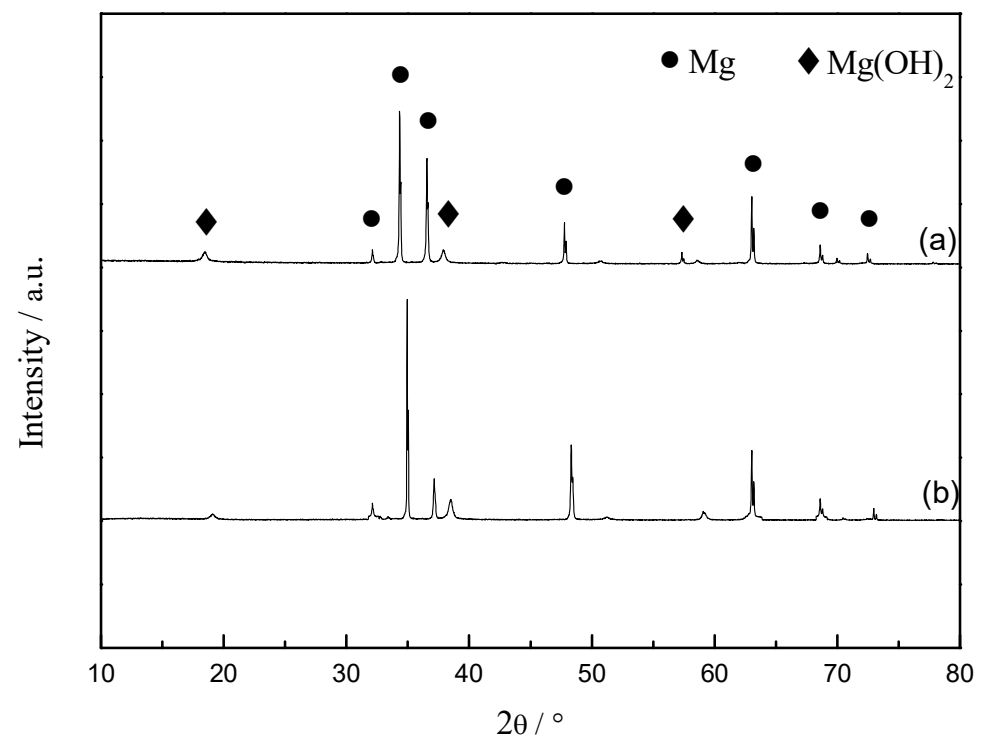

Figure 9. X-ray diffractometer (XRD) spectra of typical samples: (a) CD + HT; (b) CD.

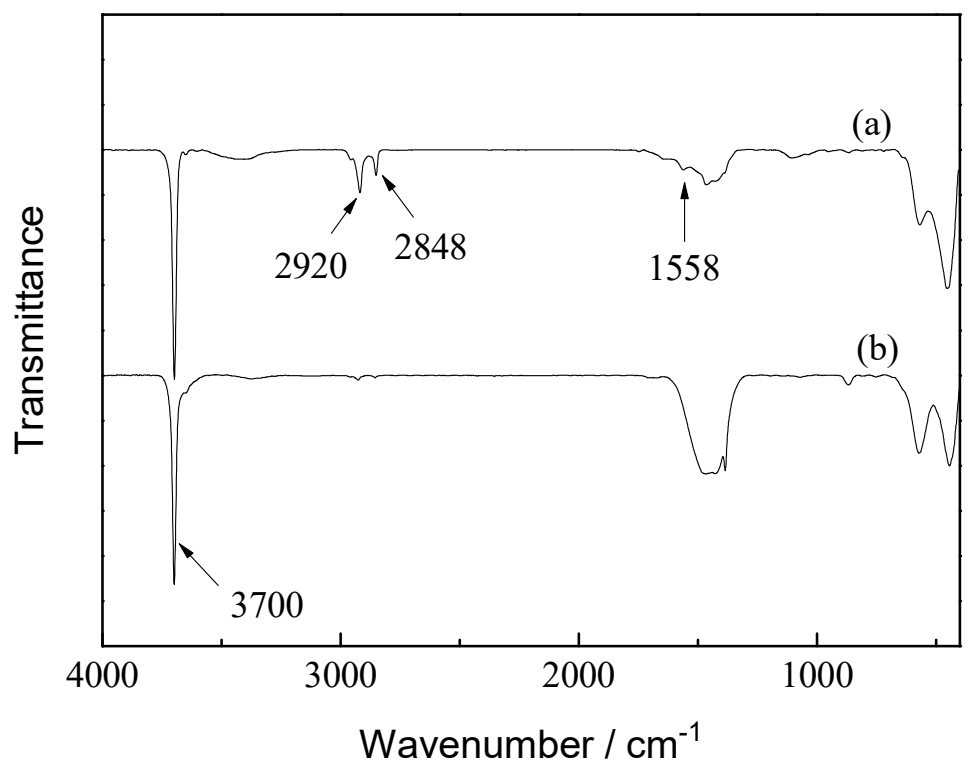

Figure 10. Fourier transform infrared spectrometer (FT-IR) spectra of typical sample surfaces: (a) CD + HT; (b) CD.

\section{Conclusions}

In summary, superhydrophobic and highly corrosion resistant surfaces were manufactured by surface modification of $\mathrm{Mg}(\mathrm{OH})_{2}$ coating prepared by cathodic deposition with ethanol solution of stearic acid. The following conclusions are drawn:

- Superhydrophobic surface with admirable $151.5^{\circ}$ water static contact angle and $1^{\circ}$ rolling angle could be obtained on $\mathrm{Mg}$ alloy substrate by $\mathrm{CD}+\mathrm{HT}$ composite treatment in which $\mathrm{Mg}(\mathrm{OH})_{2}$ film was prepared by cathodic deposition firstly and then a simple immersion was done in a low-concentration ethanol solution of stearic acid for a short period. The optimal surface modifying parameters were $5 \mathrm{~g} / \mathrm{L}$ of stearic acid at $80^{\circ} \mathrm{C}$ for $1 \mathrm{~h}$ of immersion. 
- Only CD/HT composite modification could realize surface superhydrophobization of Mg alloy, whereas single cathodic deposition or single hydrophobic treatment could not.

- SEM observation showed that $\mathrm{Mg}(\mathrm{OH})_{2}$ coating modified by stearic acid consisted of blade-like structures with micro/nano scale sizes. This kind of micro/nano structures were interlaced to form a large number of voids, which effectively inhibited surface contact with water and fluids and significantly improved the corrosion resistance of Mg alloy.

- The FT-IR spectrum test results showed that new $-\mathrm{CH}_{2}$ - peaks appeared in the infrared spectrum of the CD + HT sample, indicating that stearic acid had reacted with the $\mathrm{Mg}(\mathrm{OH})_{2}$ film in a self-assembled or chemically bonded manner.

Author Contributions: Conceptualization, Y.Z. and H.C.; Software, Z.W. and H.H.; Validation, H.C., Z.W. and H.H.; Formal Analysis, Y.Z.; Investigation, H.C. and Z.W.; Resources, Y.Z.; Data curation, H.C. and H.H.; Writing—original draft preparation, H.C.; Writing—review and editing, Y.Z., Z.W. and H.H.; Supervision, Y.Z.; Project Administration, Y.Z. and H.H.; Funding Acquisition, Y.Z.

Funding: Projects supported by National Natural Science Foundation of China (51533003) and CRRC Dalian Institute Co., Ltd. (2018440002000988).

Conflicts of Interest: The authors declare no conflict of interest.

\section{References}

1. Wang, Z.W.; Li, Q.; She, Z.X.; Chen, F.N.; Li, L.Q.; Zhang, X.X.; Zhang, P. Facile and fast fabrication of superhydrophobic surface on magnesium alloy. Appl. Surf. Sci. 2013, 271, 182-192. [CrossRef]

2. Song, G.L. Corrosion and Protection of Mg Alloy, 1st ed.; Chemical Industry Press: Beijing, China, 2006. (In Chinese)

3. Xi, T.F.; Zheng, Y.F. Current situation and development and tendency of interventional medical device. Mater. China 2010, 29, 17-26. (In Chinese)

4. Tan, Y. An overview of techniques for characterizing in homogeneities in organic surface films and under film localized corrosion. Prog. Org. Coat. 2013, 76, 791-803. [CrossRef]

5. Kumar, P.S.; Sundaramurthy, J.; Zhang, X.; Mangalaraj, D.; Thavasi, V.; Ramakrishna, S. Superhydrophobic and antireflecting behavior of densely packed and size controlled ZnO nanorods. J. Alloy. Compd. 2013, 553, 375-382. [CrossRef]

6. Qian, H.C.; Li, H.Y.; Zhang, D.W. Research progress of superhydrophobic surface technologies in the field of corrosion protection. Surf. Technol. 2015, 44, 15-24.

7. Celia, E.; Darmanin, T.; Givenchy, E.T.; Amigoni, S.; Guittard, F. Recent advances in designing superhydrophobic surfaces. J. Colloid Interface Sci. 2013, 402, 1-18. [CrossRef]

8. Darmanin, T.; Guittard, F. Recent advances in the potential applications of bioinspired superhydrophobic materials. J. Mater. Chem. A 2014, 2, 16319-16359. [CrossRef]

9. Song, J.L.; Lu, Y.; Huang, S.; Liu, X.; Wu, L.B.; Xu, W.J. A simple immersion approach for fabricating superhydrophobic Mg alloy surfaces. Appl. Surf. Sci. 2013, 266, 445-450. [CrossRef]

10. Zhang, Q.; Cao, K.; Chen, F.M. Fabrication of superhydrophobic surface on metallic substrate by pulse $\mathrm{Cu}$ electroplating and silver plating. China Surf. Eng. 2015, 28, 1-8. (In Chinese)

11. Zhang, W.; Lu, P.; Qian, L. Fabrication of superhydrophobic paper surface via wax mixture coating. Chem. Eng. J. 2014, 250, 431-436. [CrossRef]

12. Zhou, M.; Pang, X.; Wei, L.; Gao, K. Insitu grown superhydrophobic Zn-Al layered double hydroxides films on magnesium alloy to improve corrosion properties. Appl. Surf. Sci. 2015, 337, 172-177. [CrossRef]

13. Shirtcliffe, N.J.; McHale, G.; Newton, M.I.; Perry, C.C. Intrinsically superhydrophobic organosilica sol-gel foams. Langmuir 2003, 19, 5626-5631. [CrossRef]

14. Yang, J.; Zhang, Z.; Men, X.; Xu, X.; Zhu, X. Reversible superhydrobicity to superhydrophilicity switching of a carbon nanotube film via alternation of UV irradiation and dark storage. Langmuir 2010, 26, 10198-10202. [CrossRef]

15. Xi, W.; Qiao, Z.; Zhu, C.; Jia, A.; Li, M. The preparation of lotus-like super-hydrophobic copper surfaces by electroplating. Appl. Surf. Sci. 2009, 255, 4836-4839. [CrossRef] 
16. Chu, F.; Wu, X. Fabrication and condensation characteristics of metallic superhydrophobic surfaces with hierarchical micro-nano structures. Appl. Surf. Sci. 2016, 371, 322-328. [CrossRef]

17. Zhang, H.M.; Wang, T. Preparation and hydrophobic properties of the micro-nano structure of butterfly wing surface. China Surf. Eng. 2014, 27, 131-136.

18. Chen, Z.; Hao, L.; Chen, C. A fast electrodeposition method for fabrication of lanthanum superhydrophobic surface with hierarchical micro-nanostructures. Colloids Surf. A. 2012, 401, 1-7. [CrossRef]

19. Huang, Y.; Sarkar, D.K.; Grant Chen, X. Superhydrophobic aluminum alloy surfaces prepared by chemical etching process and their corrosion resistance properties. Appl. Surf. Sci. 2015, 356, 1012-1024. [CrossRef]

20. Jia, S.G. Study on cathodic deposition of $\mathrm{Mg}(\mathrm{OH})_{2}$ for surface modification of $\mathrm{Mg}$ alloy. Master's Thesis, South China University of Technology, Guangzhou, China, 2015.

21. Zhong, Y.; Hu, J.; Zhang, Y.; Tang, S. The one-step electroposition of superhydrophobic surface on AZ31 magnesium alloy and its time-dependence corrosion resistance in $\mathrm{NaCl}$ solution. Appl. Surf. Sci. 2018, 427, 1193-1201. [CrossRef]

22. Zhang, Y.; Tang, S.; Hu, J.; Lin, T. Formation mechanism and corrosion resistance of the hydrophobic coating on anodized magnesium. Corros. Sci. 2016, 111, 334-343. [CrossRef]

23. Gao, R.; Liu, Q.; Wang, J.; Zhang, X.; Yang, W.; Liu, J.; Liu, L. Fabrication of fibrous szaibelyite with hierarchical structure superhydrophobic coating on AZ31 magnesium alloy for corrosion protection. Chem. Eng. J. 2014, 241, 352-359. [CrossRef]

24. Liu, Y.; Yin, X.; Zhang, J. An electro-deposition process for fabrication of biomimetic super-hydrophobic surface and its corrosion resistance on Mg alloy. Electrochem. Acta 2014, 125, 395-403. [CrossRef]

25. Zhu, Y.L.; Fan, W.B.; Feng, L.B. Anti-adhesion and corrosion resistance of superhydrophobic Mg alloy surface. J. Mater. Eng. 2016, 44, 66-70.

26. Han, M.; Go, S.; Ahn, Y. Fabrication of superhydrophobic surface on magnesium substrate by chemical etching. Bull. Korean Chem. Soc. 2012, 33, 1363-1366. [CrossRef]

27. Ishizaki, T.; Sakamoto, M. Facile formation of biomimetic color-tuned superhydrophobic magnesium alloy with corrosion resistance. Langmuir 2011, 27, 2375-2381. [CrossRef]

28. ASTM D3359-09 Standard Test Methods for Measuring Adhesion by Tape Test; ASTM International: West Conshohocken, PA, USA, 2009.

(C) 2019 by the authors. Licensee MDPI, Basel, Switzerland. This article is an open access article distributed under the terms and conditions of the Creative Commons Attribution (CC BY) license (http://creativecommons.org/licenses/by/4.0/). 